
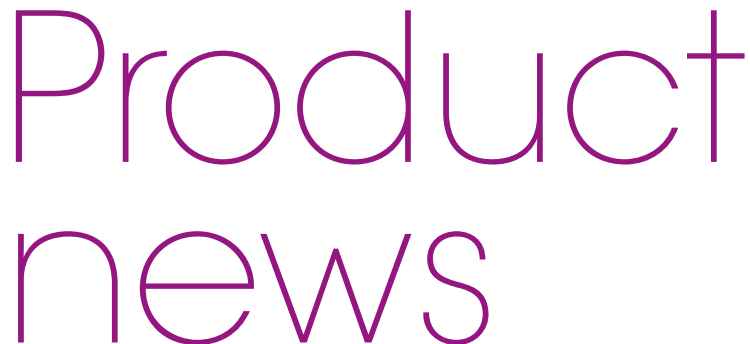

Product news is provided as a service to readers using text and images from the manufacturer, supplier or distributor and does not imply endorsement by BDJ Team. Normal and prudent research should be exercised before purchase or use of any product mentioned.

\title{
PRIMESCAN FOR PRECISION SCANNING
}

Are you looking for a digital intraoral scanner that boasts enhanced accuracy, usability and speed? Consider Dentsply Sirona's revolutionary new Primescan, available from dental equipment supplier, Clark Dental.

Primescan has the facility to export STL and exocad files to your preferred laboratory, or - in combination with the latest CEREC systems - to design restorations and produce sophisticated CEREC guides for dental implants. When combined with CEREC milling units, Primescan is able to effectively support full chairside workflows for single-visit dentistry.

Featuring advanced smart pixel sensors and a state-of-the-art dynamic lens, Primescan is able to process over 1 million $3 \mathrm{D}$ points per second, producing
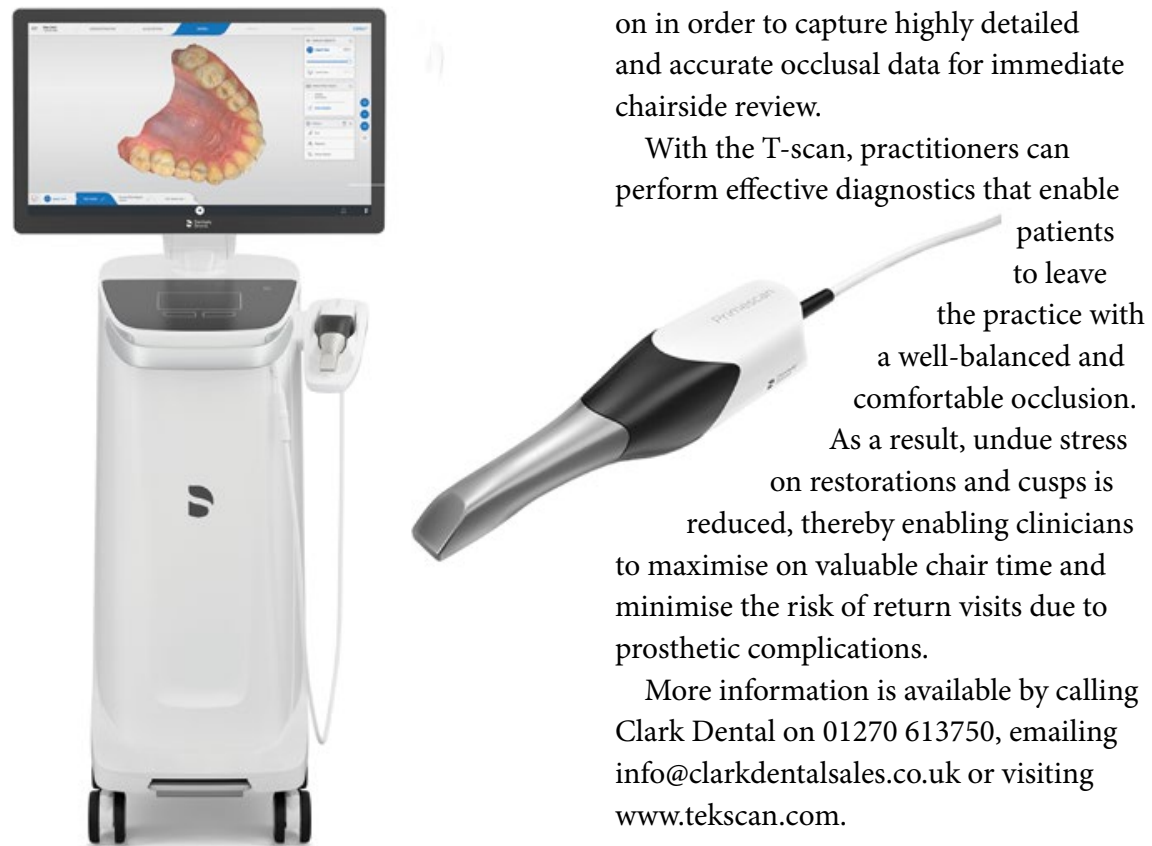

to maximise on valuable chair time and minimise the risk of return visits due to prosthetic complications.

More information is available by calling Clark Dental on 01270 613750, emailing info@clarkdentalsales.co.uk or visiting www.tekscan.com. highly accurate, photorealistic images of the oral cavity.

With the ability to scan various denta and glass - Primescan's increased field of view enables you to visualise larger areas with outstanding precision and minimal movement. High-resolution scans are instantly displayed on the touchscreen Centre.

Primescan supports a wide range of dental procedures, from single-tooth to full-arch treatment plans. Contact Clark Dental today on 01268733146 for a Primescan's unique features.

Also available from Clark Dental is the innovative T-scan occlusal analyser. a unique sensor that patients bite down on in order to capture highly detailed and accurate occlusal data for immediate chairside review.

With the T-scan, practitioners can perform effective diagnostics that enable

\section{ORAL CANCER SCREENING GLASSES}

Dental Advisor has rated Goccles - the oral cancer screening glasses - as their preferred product for oral cancer screening.

The organisation provides dental professionals worldwide with concise, accurate, and objective information on a product's performance, combining clinical experience with laboratory data and long-term in-vivo performance of materials over time.

With an enviable rating of $92 \%$, they found the Goccles system easy to use and effective at displaying early lesions.

Several of the consultants who reviewed the product also commented on its appeal to patients, with one saying 'Patients liked that it was obvious that I was giving them an oral cancer screening and using technology. Patients perceived that they were getting a better-quality exam.'

Goccles allow the clinician to run a simple, non-invasive and painless test using the technology of fluorescence and cell-tissue autofluorescence by utilising the wavelengths emitted by curing-lights.

This allows the user to see in a clear and accurate way any anomalies of the oral cavity. The basic principle is that the auto-fluorescence of abnormal cells lining the mouth when exposed to light, differs to that seen occurring in normal cells.

Goccles glasses allow the clinician to see differences in auto-fluorescence of the tissues, with normal cells appearing green and abnormal cells dark. Goccles are distributed exclusively through Dental Sky.

More information is available by visiting https://www.dentalsky.com/goccles-oralcancer-screening-glasses.html.

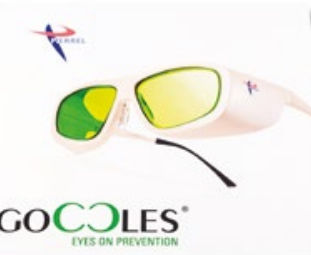

If you would like to promote your products or services direct to the dental industry in BDJ Team, call Andy May on 02078434785 or emaila.may@nature.com 